\title{
Apparatus for the Preparation of Anhydrous Titanium (III) Chloride and Titanium (III) Bromide
}

\author{
J. M. Sherfey
}

\begin{abstract}
An improved method is described for the preparation of titanium (III) chloride or titanium (III) bromide from the corresponding tetrahalide. The apparatus consists essentially of a reaction kettle, in the upper half of which is suspended a hot tungsten filament. A mixture of hydrogen and the vapor of the refluxing tetrahalide reacts at the surface of the filament, and the resulting solid product collects at the bottom of the vessel.
\end{abstract}

The laboratory preparation of anhydrous titanium (III) chloride has been described by several workers. The method described by Georges and Stahler ${ }^{1}$ has been perhaps the most satisfactory. A stream of hydrogen is saturated with titanium (IV) chloride, and the mixture is passed through an annular space formed by two concentric tubes. The inner wall is electrically heated, and the outer wall is cooled by a water jacket. The mixture reacts at the hot surface, and the resulting trichloride collects on the cold surface. It was found that the rate of production of titanium (III) chloride in the apparatus described was only a few grams per hour and that the yield, particularly with regard to the hydrogen used, was very poor. The product was contaminated with tetrachloride, especially if the temperature of the tetrachloride saturator was high.

The apparatus described below, utilizing the same reaction $\left(2 \mathrm{TiCl}_{4}+\mathrm{H}_{2} \rightleftharpoons 2 \mathrm{TiCl}_{3}+2 \mathrm{HCl}\right)$, has been found very satisfactory, and 150 to $200 \mathrm{~g}$ can be produced in 1 day. The product is about 98 percent pure and can be further purified.

The essential parts of the apparatus are shown in figure 1 . The 4-liter reaction kettle, $\mathrm{A}$, has a flanged top with four female standard taper openings, one $(34 / 45)$ centrally located with three $(24 / 40)$ joints symmetrically spaced around it. For simplicity these are shown in the drawing as if they were in a straight line.

The center joint, B, of the reaction kettle is fitted with a chimney, $\mathrm{C}$, in the top of which is a rubber stopper, D. Two tungsten rods, E, about $6 \mathrm{~mm}$ in diameter, pass through this stopper and support a tungsten filament, $\mathrm{F}$. The diameter and length of this filament are fixed by the power supply available. A filament $1 \mathrm{~mm}$ in diameter and $30 \mathrm{~cm}$ long requires 38 amperes at 8.6 volts to heat it to $1,000^{\circ}$ to $1,100^{\circ} \mathrm{C}$, a satisfactory temperature range. Thinner filaments should not be used because they are apt to burn out during a run.

A run is started by flushing the entire apparatus

1 Ber. deut. chem. Ges. 42, 3200 (1909); see also, Stahler and Bachran, Ber. deut. chem. Ges. 44, 2906 (1911).

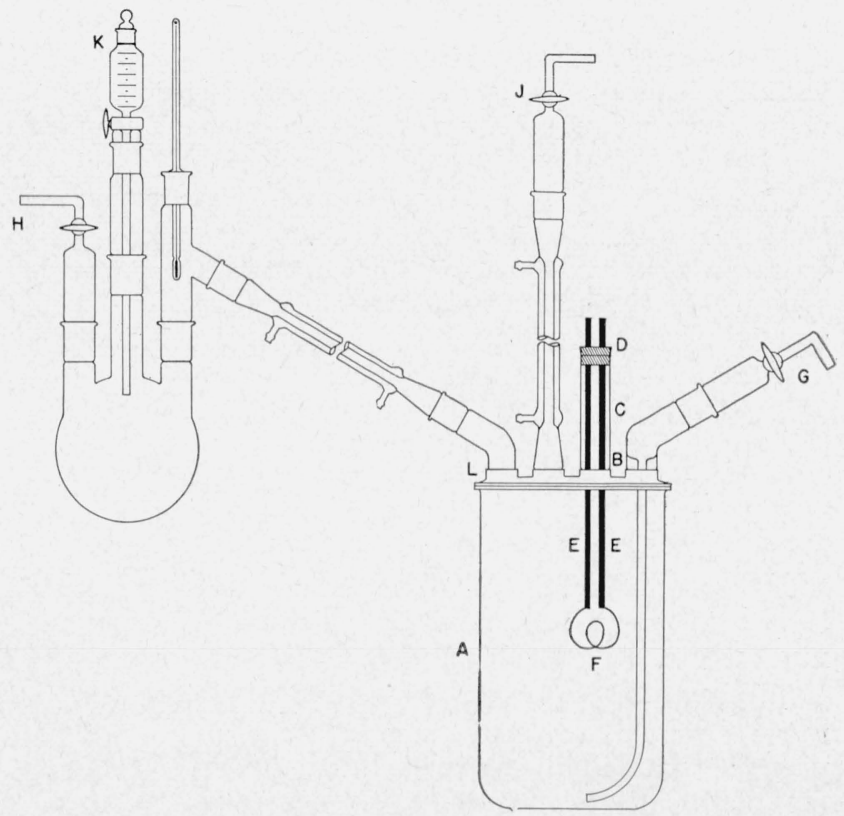

FIGURE 1. Apparatus for the preparation of anhydrous titanium (III) chloride and titanium (III) bromide.

A, Pyrex reaction kettle; B, central standard taper joint of reaction kettle; C, chimney; D, rubber stopper; E, 6-mm tungsten rods; F, 1-mm tungsten filament; G, hydrogen inlet; $\mathrm{H}$, initial hydrogen outlet; J, final hydrogen outlets; $\mathrm{K}$, burette.

with dry oxygen-free hydrogen, purified by passing it over hot copper turnings and then over a desiccant such as magnesium perchlorate. After purification the hydrogen should pass through copper tubing or, if necessary, a minimum length of sound rubber tubing. The purified hydrogen is introduced at $\mathrm{G}$ and passes through the distillation assembly and out through the stopcock, $\mathrm{H}$, until all the air has been removed. This will also remove moisture and thus prevent the formation of hydrolysis products.

The hydrogen stream is continued while stopcock $\mathrm{H}$ is closed and that at $\mathrm{J}$ is opened. About 1,500 $\mathrm{ml}$ of titanium (IV) chloride is run into the distilling flask through the burette, $\mathrm{K}$, and distillation is started. About 1,000 to $1,500 \mathrm{ml}$ is distilled over into the reaction kettle. The distilling apparatus can then be removed from the reaction kettle and the opening, L, quickly stoppered. The hydrogen 
stream should be very rapid during this operation.

A hemispherical heating mantle is now used to reflux the tetrachloride over the tungsten filament. A heating mantle that entirely encloses the reaction kettle must not be used, as this will cause the tetrachloride to reflux too rapidly and flood the condenser. This situation is detrimental because, after the reduction is started, solid trichloride will be carried out of the apparatus by the hydrogen stream. Most, if not all, of the tetrachloride should be condensed on the walls and top of the reaction flask. Jets of air directed against the upper part of the flask will aid in this.

When the boiling of the tetrachloride has reached equilibrium, the filament is turned on and brought to a bright red heat. Reduction begins with the formation of a dark purple smoke of titanium (III) chloride which collects on the walls of the reaction vessel. Most of these particles are washed down to the bottom by the refluxing tetrachloride.

If for any reason the vessel is cooled, for instance by turning off the filament, the hydrogen flow must be momentarily increased to prevent influx of air as a result of the partial vacuum formed. This influx would cause hydrolysis and oxidation. A length of rubber tubing on outlet $J$ minimizes this danger.

The reaction is continued until the tetrachloride no longer refluxes freely down the sides of the vessel. The filament is then turned off, and the whole reaction kettle is heated to drive off the residual tetrachloride, which tends to be absorbed in the cake of solid trichloride. To condense and remove the tetrachloride, a still head and horizontal condenser must be substituted for the reflux condenser.

The hydrogen stream is continued while the apparatus cools. The flask should be opened and the product bottled in an inert atmosphere. Titanium (III) chloride thus formed often ignites spontaneously when exposed to moist air and invariably does so when gently heated.

In a typical run, 1 liter $(1,700 \mathrm{~g})$ of titanium (IV) chloride was distilled into the reaction flask and yielded $154 \mathrm{~g}$ of titanium (III) chloride, a conversion of 10 percent. Based on the tetrachloride consumed, the yield of trichloride was about 90 percent.
Analysis of the product gave

\begin{tabular}{|c|c|c|c|}
\hline & $\mathrm{Ti}$ & $\mathrm{Cl}$ & Reducing power \\
\hline $\begin{array}{l}\text { Found (percent) } \\
\text { Calculated (percent) }\end{array}$ & $\begin{array}{l}\text { 31. } 9 \\
\text { 31. } 0\end{array}$ & $\begin{array}{l}\text { 67. } 6 \\
68.9\end{array}$ & $\begin{array}{l}\text { 97. } 2,98.7 \\
\text { 100.0 }\end{array}$ \\
\hline
\end{tabular}

The reducing power was determined by titration with potassium dichromate. The crude product was heated in a vacuum at $200^{\circ} \mathrm{C}$, and a small quantity of a volatile impurity was driven off. After this treatment a reduction value of 101.5 percent was obtained, indicating the presence of a small amount of titanium (II) chloride.

It is possible to obtain a much higher percentage conversion, particularly if the whole flask is heated, but two factors must be borne in mind. First, a solid reaction mass at the bottom is no longer being stirred by its own ebullition, and second, the solid cake on the sides, if no longer washed down by the refluxing tetrachloride, tends to build out toward the filament. Either of these factors could result in localized overheating and consequent disproportionation of trichloride into dichloride and tetrachloride.

The rate of formation of titanium (III) chloride is largely determined by the size of the filament. This limitation could be circumvented if a sheet of tungsten, preferably in the shape of a cylinder, were heated by induction.

Titanium (III) bromide has been prepared in the same apparatus. As the tetrabromide is solid at room temperature, hot water or steam is used as a coolant for the condensers (instead of the cold water used in the preparation of trichloride). The tetrabromide is poured hot into the distilling flask and allowed to solidify before flushing with hydrogen. The yield and purity of the product are very similar to those reported for the trichloride.

In some runs a multiple collecting device was used, which made it possible to discard the first fraction of distilled tetrachloride without opening the apparatus. It has not been shown that this is either necessary or beneficial.

Washington, November 17, 1950 